\title{
GlossBERT: BERT for Word Sense Disambiguation with Gloss Knowledge
}

\author{
Luyao Huang, Chi Sun, Xipeng Qiu, Xuanjing Huang \\ Shanghai Key Laboratory of Intelligent Information Processing, Fudan University \\ School of Computer Science, Fudan University \\ 825 Zhangheng Road, Shanghai, China \\ \{1yhuang18, sunc17, xpqiu, xjhuang\}@fudan.edu.cn
}

\begin{abstract}
Word Sense Disambiguation (WSD) aims to find the exact sense of an ambiguous word in a particular context. Traditional supervised methods rarely take into consideration the lexical resources like WordNet, which are widely utilized in knowledge-based methods. Recent studies have shown the effectiveness of incorporating gloss (sense definition) into neural networks for WSD. However, compared with traditional word expert supervised methods, they have not achieved much improvement. In this paper, we focus on how to better leverage gloss knowledge in a supervised neural WSD system. We construct context-gloss pairs and propose three BERT-based models for WSD. We fine-tune the pre-trained BERT model and achieve new state-of-the-art results on WSD task.
\end{abstract}

\section{Introduction}

Word Sense Disambiguation (WSD) is a fundamental task and long-standing challenge in Natural Language Processing (NLP), which aims to find the exact sense of an ambiguous word in a particular context (Navigli, 2009). Previous WSD approaches can be grouped into two main categories: knowledge-based and supervised methods.

Knowledge-based WSD methods rely on lexical resources like WordNet (Miller, 1995) and usually exploit two kinds of lexical knowledge. The gloss, which defines a word sense meaning, is first utilized in Lesk algorithm (Lesk, 1986) and then widely taken into account in many other approaches (Banerjee and Pedersen, 2002; Basile et al., 2014). Besides, structural properties of semantic graphs are mainly used in graph-based algorithms (Agirre et al., 2014; Moro et al., 2014).

Traditional supervised WSD methods (Zhong and Ng, 2010; Shen et al., 2013; Iacobacci et al.,

\footnotetext{
${ }^{*}$ Corresponding author.
}

2016) focus on extracting manually designed features and then train a dedicated classifier (word expert) for every target lemma.

Although word expert supervised WSD methods perform better, they are less flexible than knowledge-based methods in the all-words WSD task (Raganato et al., 2017a). Recent neural-based methods are devoted to dealing with this problem. Kågebäck and Salomonsson (2016) present a supervised classifier based on Bi-LSTM, which shares parameters among all word types except the last layer. Raganato et al. (2017a) convert WSD task to a sequence labeling task, thus building a unified model for all polysemous words. However, neither of them can totally beat the best word expert supervised methods.

More recently, Luo et al. (2018b) propose to leverage the gloss information from WordNet and model the semantic relationship between the context and gloss in an improved memory network. Similarly, Luo et al. (2018a) introduce a (hierarchical) co-attention mechanism to generate co-dependent representations for the context and gloss. Their attempts prove that incorporating gloss knowledge into supervised WSD approach is helpful, but they still have not achieved much improvement, because they may not make full use of gloss knowledge.

In this paper, we focus on how to better leverage gloss information in a supervised neural WSD system. Recently, the pre-trained language models, such as ELMo (Peters et al., 2018) and BERT (Devlin et al., 2018), have shown their effectiveness to alleviate the effort of feature engineering. Especially, BERT has achieved excellent results in question answering (QA) and natural language inference (NLI). We construct context-gloss pairs from glosses of all possible senses (in WordNet) of the target word, thus treating WSD task as a sentence-pair classification problem. We fine- 


\begin{tabular}{|c|c|c|}
\hline \multicolumn{3}{|l|}{$\begin{array}{l}\text { Sentence with four targets: } \\
\text { Your research stopped when a convenient assertion could be made. }\end{array}$} \\
\hline Context-Gloss Pairs of the target word [research] & Label & Sense Key \\
\hline [CLS ] Your research ... [SEP ] systematic investigation to ... [SEP ] & Yes & research\%1:04:00:: \\
\hline [CLS ] Your research ... [SEP ] a search for knowledge [SEP ] & No & research\% 1:09:00:: \\
\hline [CLS ] Your research ... [SEP ] inquire into [SEP ] & No & research\%2:31:00:: \\
\hline$[\mathrm{CLS}]$ Your research ... [SEP $]$ attempt to find out in a ... [SEP ] & No & research\%2:32:00:: \\
\hline Context-Gloss Pairs with weak supervision of the target word [research] & Label & Sense Key \\
\hline [CLS ] Your "research" ... [ SEP ] research: systematic investigation to ... [ SEP ] & Yes & research\% 1:04:00:: \\
\hline [CLS ] Your "research"... [SEP ] research: a search for knowledge [SEP ] & No & research\% 1:09:00:: \\
\hline [CLS ] Your "research" ... [SEP ] research: inquire into [SEP ] & No & research\%2:31:00:: \\
\hline [CLS ] Your "research"... [ SEP ] research: attempt to find out in a ... [SEP ] & No & research\%2:32:00:: \\
\hline
\end{tabular}

Table 1: The construction methods. The sentence is taken from SemEval-2007 WSD dataset. The ellipsis "..." indicates the remainder of the sentence or the gloss.

tune the pre-trained BERT model and achieve new state-of-the-art results on WSD task. In particular, our contribution is two-fold:

1. We construct context-gloss pairs and propose three BERT-based models for WSD.

2. We fine-tune the pre-trained BERT model, and the experimental results on several English allwords WSD benchmark datasets show that our approach significantly outperforms the state-of-theart systems.

\section{Methodology}

In this section, we describe our method in detail.

\subsection{Task Definition}

In WSD, a sentence $s$ usually consists of a series of words: $\left\{w_{1}, \cdots, w_{m}\right\}$, and some of the words $\left\{w_{i_{1}}, \cdots, w_{i_{k}}\right\}$ are targets $\left\{t_{1}, \cdots, t_{k}\right\}$ need to be disambiguated. For each target $t$, its candidate senses $\left\{c_{1}, \cdots, c_{n}\right\}$ come from entries of its lemma in a pre-defined sense inventory (usually WordNet). Therefore, WSD task aims to find the most suitable entry (symbolized as unique sense key) for each target in a sentence. See a sentence example in Table 1.

\subsection{BERT}

BERT (Devlin et al., 2018) is a new language representation model, and its architecture is a multilayer bidirectional Transformer encoder. BERT model is pre-trained on a large corpus and two novel unsupervised prediction tasks, i.e., masked language model and next sentence prediction tasks are used in pre-training. When incorporating BERT into downstream tasks, the fine-tuning procedure is recommended. We fine-tune the pretrained BERT model on WSD task.
BERT(Token-CLS) Since every target in a sentence needs to be disambiguated to find its exact sense, WSD task can be regarded as a token-level classification task. To incorporate BERT to WSD task, we take the final hidden state of the token corresponding to the target word (if more than one token, we average them) and add a classification layer for every target lemma, which is the same as the last layer of the Bi-LSTM model (Kågebäck and Salomonsson, 2016).

\subsection{GlossBERT}

BERT can explicitly model the relationship of a pair of texts, which has shown to be beneficial to many pair-wise natural language understanding tasks. In order to fully leverage gloss information, we propose GlossBERT to construct context-gloss pairs from all possible senses of the target word in WordNet, thus treating WSD task as a sentencepair classification problem.

We describe our construction method with an example (See Table 1). There are four targets in this sentence, and here we take target word research as an example:

Context-Gloss Pairs The sentence containing target words is denoted as context sentence. For each target word, we extract glosses of all $N$ possible senses (here $N=4$ ) of the target word (research) in WordNet to obtain the gloss sentence. [CLS] and [SEP] marks are added to the context-gloss pairs to make it suitable for the input of BERT model. A similar idea is also used in aspect-based sentiment analysis (Sun et al., 2019).

\section{Context-Gloss Pairs with Weak Supervision} Based on the previous construction method, we add weak supervised signals to the context-gloss 


\begin{tabular}{|c|c|c|c|c|c|}
\hline Dataset & Total & Noun & Verb & Adj & Adv \\
\hline SemCor & 226036 & 87002 & 88334 & 31753 & 18947 \\
\hline SE2 & 2282 & 1066 & 517 & 445 & 254 \\
\hline SE3 & 1850 & 900 & 588 & 350 & 12 \\
\hline SE07 & 455 & 159 & 296 & 0 & 0 \\
\hline SE13 & 1644 & 1644 & 0 & 0 & 0 \\
\hline SE15 & 1022 & 531 & 251 & 160 & 80 \\
\hline
\end{tabular}

Table 2: Statistics of the different parts of speech annotations in English all-words WSD datasets.

pairs (see the highlighted part in Table 1). The signal in the gloss sentence aims to point out the target word, and the signal in the context sentence aims to emphasize the target word considering the situation that a target word may occur more than one time in the same sentence.

Therefore, each target word has $N$ context-gloss pair training instances (label $\in\{y e s, n o\}$ ). When testing, we output the probability of label =yes of each context-gloss pair and choose the sense corresponding to the highest probability as the prediction label of the target word. We experiment with three GlossBERT models:

GlossBERT(Token-CLS) We use context-gloss pairs as input. We highlight the target word by taking the final hidden state of the token corresponding to the target word (if more than one token, we average them) and add a classification layer (label $\in\{y e s$, no $\}$ ).

GlossBERT(Sent-CLS) We use context-gloss pairs as input. We take the final hidden state of the first token [CLS] as the representation of the whole sequence and add a classification layer (label $\in\{y e s, n o\}$ ), which does not highlight the target word.

GlossBERT(Sent-CLS-WS) We use contextgloss pairs with weak supervision as input. We take the final hidden state of the first token [CLS ] and add a classification layer (label $\in\{$ yes, no $\}$ ), which weekly highlight the target word by the weak supervision.

\section{Experiments}

\subsection{Datasets}

The statistics of the WSD datasets are shown in Table 2.

Training Dataset Following previous work (Luo et al., 2018a,b; Raganato et al., 2017a,b; Iacobacci et al., 2016; Zhong and Ng, 2010), we choose SemCor3.0 as training corpus, which is the largest corpus manually annotated with WordNet sense for WSD.

Evaluation Datasets We evaluate our method on several English all-words WSD datasets. For a fair comparison, we use the benchmark datasets proposed by Raganato et al. (2017b) which include five standard all-words fine-grained WSD datasets from the Senseval and SemEval competitions: Senseval-2 (SE2), Senseval-3 (SE3), SemEval-2007 (SE07), SemEval-2013 (SE13) and SemEval-2015 (SE15). Following Luo et al. (2018a), Luo et al. (2018b) and Raganato et al. (2017a), we choose SE07, the smallest among these test sets, as the development set.

WordNet Since Raganato et al. (2017b) map all the sense annotations in these datasets from their original versions to WordNet 3.0, we extract word sense glosses from WordNet 3.0.

\subsection{Settings}

We use the pre-trained uncased BERT $\mathrm{BASE}_{\mathrm{B}}$ model $^{1}$ for fine-tuning, because we find that BERT $_{\text {LARGE }}$ model performs slightly worse than BERT $_{\text {BASE }}$ in this task. The number of Transformer blocks is 12 , the number of the hidden layer is 768 , the number of self-attention heads is 12 , and the total number of parameters of the pre-trained model is $110 \mathrm{M}$. When fine-tuning, we use the development set (SE07) to find the optimal settings for our experiments. We keep the dropout probability at 0.1 , set the number of epochs to 4 . The initial learning rate is $2 \mathrm{e}-5$, and the batch size is 64 .

\subsection{Results}

Table 3 shows the performance of our method on the English all-words WSD benchmark datasets. We compare our approach with previous methods.

The first block shows the MFS baseline, which selects the most frequent sense in the training corpus for each target word.

The second block shows two knowledge-based

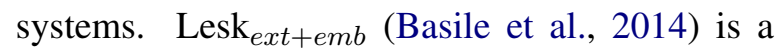
variant of Lesk algorithm (Lesk, 1986) by calculating the gloss-context overlap of the target word. Babelfy (Moro et al., 2014) is a unified graphbased approach which exploits the semantic network structure from BabelNet.

\footnotetext{
${ }^{1}$ https://storage.googleapis.com/bert_models/2018_10_18/ uncased_L-12_H-768_A-12.zip
} 


\begin{tabular}{|c|c|c|c|c|c|c|c|c|c|c|}
\hline & Dev & \multicolumn{4}{|c|}{ Test Datasets } & \multicolumn{5}{|c|}{ Concatenation of Test Datasets } \\
\hline System & SE07 & SE2 & SE3 & SE13 & SE15 & Noun & Verb & Adj & Adv & All \\
\hline MFS baseline & 54.5 & 65.6 & 66.0 & 63.8 & 67.1 & 67.7 & 49.8 & 73.1 & 80.5 & 65.5 \\
\hline Lesk $_{e x t+e m b}$ & 56.7 & 63.0 & 63.7 & 66.2 & 64.6 & 70.0 & 51.1 & 51.7 & 80.6 & 64.2 \\
\hline Babelfy & 51.6 & 67.0 & 63.5 & 66.4 & 70.3 & 68.9 & 50.7 & 73.2 & 79.8 & 66.4 \\
\hline IMS & 61.3 & 70.9 & 69.3 & 65.3 & 69.5 & 70.5 & 55.8 & 75.6 & 82.9 & 68.9 \\
\hline $\mathrm{IMS}_{+e m b}$ & 62.6 & 72.2 & 70.4 & 65.9 & 71.5 & 71.9 & 56.6 & 75.9 & 84.7 & 70.1 \\
\hline Bi-LSTM & - & 71.1 & 68.4 & 64.8 & 68.3 & 69.5 & 55.9 & 76.2 & 82.4 & 68.4 \\
\hline $\mathrm{Bi}_{-} \mathrm{LSTM}_{+a t t .+L E X+P O S}$ & 64.8 & 72.0 & 69.1 & 66.9 & 71.5 & 71.5 & 57.5 & 75.0 & 83.8 & 69.9 \\
\hline $\mathrm{GAS}_{\text {ext }}$ (Linear) & - & 72.4 & 70.1 & 67.1 & 72.1 & 71.9 & 58.1 & 76.4 & 84.7 & 70.4 \\
\hline $\mathrm{GAS}_{\text {ext }}$ (Concatenation) & - & 72.2 & 70.5 & 67.2 & 72.6 & 72.2 & 57.7 & 76.6 & 85.0 & 70.6 \\
\hline $\mathrm{CAN}^{s}$ & - & 72.2 & 70.2 & 69.1 & 72.2 & 73.5 & 56.5 & 76.6 & 80.3 & 70.9 \\
\hline HCAN & - & 72.8 & 70.3 & 68.5 & 72.8 & 72.7 & 58.2 & 77.4 & 84.1 & 71.1 \\
\hline BERT(Token-CLS) & 61.1 & 69.7 & 69.4 & 65.8 & 69.5 & 72.0 & 57.8 & 73.5 & 84.4 & 68.6 \\
\hline GlossBERT(Sent-CLS) & 69.2 & 76.5 & 73.4 & 75.1 & 79.5 & 79.1 & 65.4 & 79.3 & 84.8 & 75.8 \\
\hline GlossBERT(Token-CLS) & 71.9 & 77.0 & 75.4 & 74.6 & 79.3 & 78.8 & 66.8 & 79.9 & 85.0 & 76.3 \\
\hline GlossBEF & 72.5 & 77.7 & 75.2 & 76.1 & 80.4 & 79.8 & 67.1 & 79.6 & 87.4 & 77.0 \\
\hline
\end{tabular}

Table 3: F1-score (\%) for fine-grained English all-words WSD on the test sets in the framework of Raganato et al. (2017b) (including the development set SE07). Bold font indicates best systems. The five blocks list the MFS baseline, two knowledge-based systems, two traditional word expert supervised systems, six recent neural-based systems and our systems, respectively. Results in first three blocks come from Raganato et al. (2017b), and others from the corresponding papers.

The third block shows two word expert traditional supervised systems. IMS (Zhong and Ng, 2010) is a flexible framework which trains SVM classifiers and uses local features. And IMS $+e m b$ (Iacobacci et al., 2016) is the best configuration of the IMS framework, which also integrates word embeddings as features.

The fourth block shows several recent neuralbased methods. Bi-LSTM (Kågebäck and Salomonsson, 2016) is a baseline for neural models. Bi-LSTM + att. $+L E X+P O S$ (Raganato et al., 2017a) is a multi-task learning framework for WSD, POS tagging, and LEX with self-attention mechanism, which converts WSD to a sequence

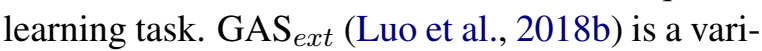
ant of GAS which is a gloss-augmented variant of the memory network by extending gloss knowledge. $\mathrm{CAN}^{s}$ and HCAN (Luo et al., 2018a) are sentence-level and hierarchical co-attention neural network models which leverage gloss knowledge.

In the last block, we report the performance of our method. BERT(Token-CLS) is our baseline, which does not incorporate gloss information, and it performs slightly worse than previous traditional supervised methods and recent neural-based methods. It proves that directly using BERT cannot obtain performance growth. The other three methods outperform other models by a substantial margin, which proves that the improvements come from leveraging BERT to better exploit gloss information. It is worth noting that our method achieves significant improvements in SE07 and Verb over previous methods, which have the highest ambi- guity level among all datasets and all POS tags respectively according to Raganato et al. (2017b).

Moreover, GlossBERT(Token-CLS) performs better than GlossBERT(Sent-CLS), which proves that highlighting the target word in the sentence is important. However, the weakly highlighting method GlossBERT(Sent-CLS-WS) performs best in most circumstances, which may result from its combination of the advantages of the other two methods.

\subsection{Discussion}

There are two main reasons for the great improvements of our experimental results. First, we construct context-gloss pairs and convert WSD problem to a sentence-pair classification task which is similar to NLI tasks and train only one classifier, which is equivalent to expanding the corpus. Second, we leverage BERT (Devlin et al., 2018) to better exploit the gloss information. BERT model shows its advantage in dealing with sentence-pair classification tasks by its amazing improvement on QA and NLI tasks. This advantage comes from both of its two novel unsupervised prediction tasks.

Compared with traditional word expert supervised methods, our GlossBERT shows its effectiveness to alleviate the effort of feature engineering and does not require training a dedicated classifier for every target lemma. Up to now, it can be said that the neural network method can totally beat the traditional word expert method. Compared with recent neural-based methods, our so- 
lution is more intuitive and can make better use of gloss knowledge. Besides, our approach demonstrates that when we fine-tune BERT on a downstream task, converting it into a sentence-pair classification task may be a good choice.

\section{Conclusion}

In this paper, we seek to better leverage gloss knowledge in a supervised neural WSD system. We propose a new solution to WSD by constructing context-gloss pairs and then converting WSD to a sentence-pair classification task. We finetune the pre-trained BERT model and achieve new state-of-the-art results on WSD task.

\section{Acknowledgments}

We would like to thank the anonymous reviewers for their valuable comments. The research work is supported by National Natural Science Foundation of China (No. 61751201 and 61672162), Shanghai Municipal Science and Technology Commission (16JC1420401 and 17JC1404100), Shanghai Municipal Science and Technology Major Project (No.2018SHZDZX01) and ZJLab.

\section{References}

Eneko Agirre, Oier López de Lacalle, and Aitor Soroa. 2014. Random walks for knowledge-based word sense disambiguation. Computational Linguistics, 40(1):57-84.

Satanjeev Banerjee and Ted Pedersen. 2002. An adapted lesk algorithm for word sense disambiguation using wordnet. In International conference on intelligent text processing and computational linguistics, pages 136-145. Springer.

Pierpaolo Basile, Annalina Caputo, and Giovanni Semeraro. 2014. An enhanced lesk word sense disambiguation algorithm through a distributional semantic model. In Proceedings of COLING 2014, the 25th International Conference on Computational Linguistics: Technical Papers, pages 1591-1600.

Jacob Devlin, Ming-Wei Chang, Kenton Lee, and Kristina Toutanova. 2018. Bert: Pre-training of deep bidirectional transformers for language understanding. arXiv preprint arXiv:1810.04805.

Ignacio Iacobacci, Mohammad Taher Pilehvar, and Roberto Navigli. 2016. Embeddings for word sense disambiguation: An evaluation study. In Proceedings of the 54th Annual Meeting of the Association for Computational Linguistics (Volume 1: Long Papers), volume 1, pages 897-907.
Mikael Kågebäck and Hans Salomonsson. 2016. Word sense disambiguation using a bidirectional 1stm. arXiv preprint arXiv:1606.03568.

Michael Lesk. 1986. Automatic sense disambiguation using machine readable dictionaries: how to tell a pine cone from an ice cream cone. In Proceedings of the 5th annual international conference on Systems documentation, pages 24-26. Citeseer.

Fuli Luo, Tianyu Liu, Zexue He, Qiaolin Xia, Zhifang Sui, and Baobao Chang. 2018a. Leveraging gloss knowledge in neural word sense disambiguation by hierarchical co-attention. In Proceedings of the 2018 Conference on Empirical Methods in Natural Language Processing, pages 1402-1411.

Fuli Luo, Tianyu Liu, Qiaolin Xia, Baobao Chang, and Zhifang Sui. 2018b. Incorporating glosses into neural word sense disambiguation. arXiv preprint arXiv:1805.08028.

George A Miller. 1995. Wordnet: a lexical database for english. Communications of the ACM, 38(11):3941 .

Andrea Moro, Alessandro Raganato, and Roberto Navigli. 2014. Entity linking meets word sense disambiguation: a unified approach. Transactions of the Association for Computational Linguistics, 2:231244.

Roberto Navigli. 2009. Word sense disambiguation: A survey. ACM computing surveys (CSUR), 41(2):10.

Matthew Peters, Mark Neumann, Mohit Iyyer, Matt Gardner, Christopher Clark, Kenton Lee, and Luke Zettlemoyer. 2018. Deep contextualized word representations. In Proceedings of the 2018 Conference of the North American Chapter of the Association for Computational Linguistics: Human Language Technologies, Volume 1 (Long Papers), pages 2227-2237.

Alessandro Raganato, Claudio Delli Bovi, and Roberto Navigli. 2017a. Neural sequence learning models for word sense disambiguation. In Proceedings of the 2017 Conference on Empirical Methods in Natural Language Processing, pages 1156-1167.

Alessandro Raganato, Jose Camacho-Collados, and Roberto Navigli. 2017b. Word sense disambiguation: A unified evaluation framework and empirical comparison. In Proceedings of the 15th Conference of the European Chapter of the Association for Computational Linguistics: Volume 1, Long Papers, pages 99-110.

Hui Shen, Razvan Bunescu, and Rada Mihalcea. 2013. Coarse to fine grained sense disambiguation in wikipedia. In Second Joint Conference on Lexical and Computational Semantics (* SEM), Volume 1: Proceedings of the Main Conference and the Shared Task: Semantic Textual Similarity, volume 1, pages 22-31. 
Chi Sun, Luyao Huang, and Xipeng Qiu. 2019. Utilizing bert for aspect-based sentiment analysis via constructing auxiliary sentence. In Proceedings of the 2019 Conference of the North American Chapter of the Association for Computational Linguistics: $\mathrm{Hu}$ man Language Technologies, Volume 1 (Long and Short Papers), pages 380-385.

Zhi Zhong and Hwee Tou Ng. 2010. It makes sense: A wide-coverage word sense disambiguation system for free text. In Proceedings of the ACL 2010 system demonstrations, pages 78-83. 